\title{
LOW DOSE NEUTRON LATE EFFECTS: CATARACTOGENESIS
}

\author{
Progress Report
}

for Period April 1, 1991 - December 15, 1991

Basil V. Worgul

Columbia University

New York, New York 10032

Derember, 1991

Prepared for

THE U.S. DEPARTMENT OF ENERGY AGREEMENT NO. DE-FG02-90ER61009 


\section{NOTICE}

This repurt was prepared as an account of work sponsored by the United States government. Neither the United States nor the Department of Energy nor any of their employees nor any of their contractors, sub-contractors nor their employees, make any warrantees, expressed or implied, nor assumes any legal liability of responsibility for the accuracy, completeness, or usefulness for any information, apparatus, product, or process disclosed, or represents that its use will not infringe privately cwned rights. 


\begin{abstract}
The work is formulated to resolve the uncertainty regarding the relative biological effectiveness (RBE) of low dose neutron radiation. The study exploits the fact that cataractogenesis is sensitive to the inverse dose-rate effect as has been observed with heavy ions and was an endpoint considered in the follow-up of the A-bomb survivors. The neutron radiations were initiated at the Radiological Research Accelerator facility (RARAF) of the Nevis Laboratory of Columbia University. Four week old ( \pm 1 day) rats were divided into eight dose groups each receiving single or fractionated total doses of $0.2,1.0,5.0$ and $25.0 \mathrm{cGy}$ of monoenergetic $435 \mathrm{KeV}$ neutrons. Special restraining jigs insured that the eye, at the midpoint of the lens, received the appropriate energy and dose with a relative error of $\pm 5 \%$. The fractionation regimen consisted of four exposures, each administered at three hour ( \pm 1 minute) intervals. The neutron irradiated groups are being compared to rats irradiated with $250 \mathrm{kVp}$ X-rays in doses ranging from 0.5 to 7 Gy. The animals are being examined on a biweekly basis utilizing conventional slit-lamp biomicroscopy and the Scheimpflug Slit Lamp Imaging System (Zeiss). The follow-ups, entering their second year, will continue throughout the life-span of the animals. This is essential inasmuch as given the extremely low doses which are being utilized clinically detectable opacities were not anticipated until a significant fraction of the life span has lapsed. Current data supports this contention. At this juncture cataracts in the irradiated groups are beginning to exceed control levels. While extremely preliminary, the earliest data suggests that dose protraction has a modulating effect on the neutron exposure. In the case of the X-ray in animals, significant sparing is observed with fractionation.
\end{abstract}




\section{Progress Report}

The specific aims of our proposal to the DOE were to conduct studies on rat eyes irradiated at the RARAF (Radiological Research Accelerator Facility) in the Nevis J aboratory of Columbia University, in order to:

1. Reassess and attempt to confirm the previous observations regarding the extraordinary RBE's reported for cataract induction following low dose neutron exposure.

2. Determine the effect of neutron dose fractionation on the overall course of the cataract development.

As pointed out in the original proposal, there are a variety of reasons that these studies are important and timely. Most recently a report by Otake and Schull (1990) on the DS86 data indicates that, consistent with the experimental experience with low-dose heavy ion exposure, the RBE's for cataracis in the Hiroshima exposed population increased with decreasing dose in terms of cataractogenesis. This was suggested, experimentally, for neutrons by Evans in 1948. Our earlier studies, using heavy ions, seem to agree in that ions with an LET in the range of the neutrons to which the survivors were exposed colld, in fact, exhibit RBE levels in rats consistent with the Otake and Schull estimates in humans. This position is borne out in the curve below comparing the RBE's for cataractogenesis estimated in varinus studies.

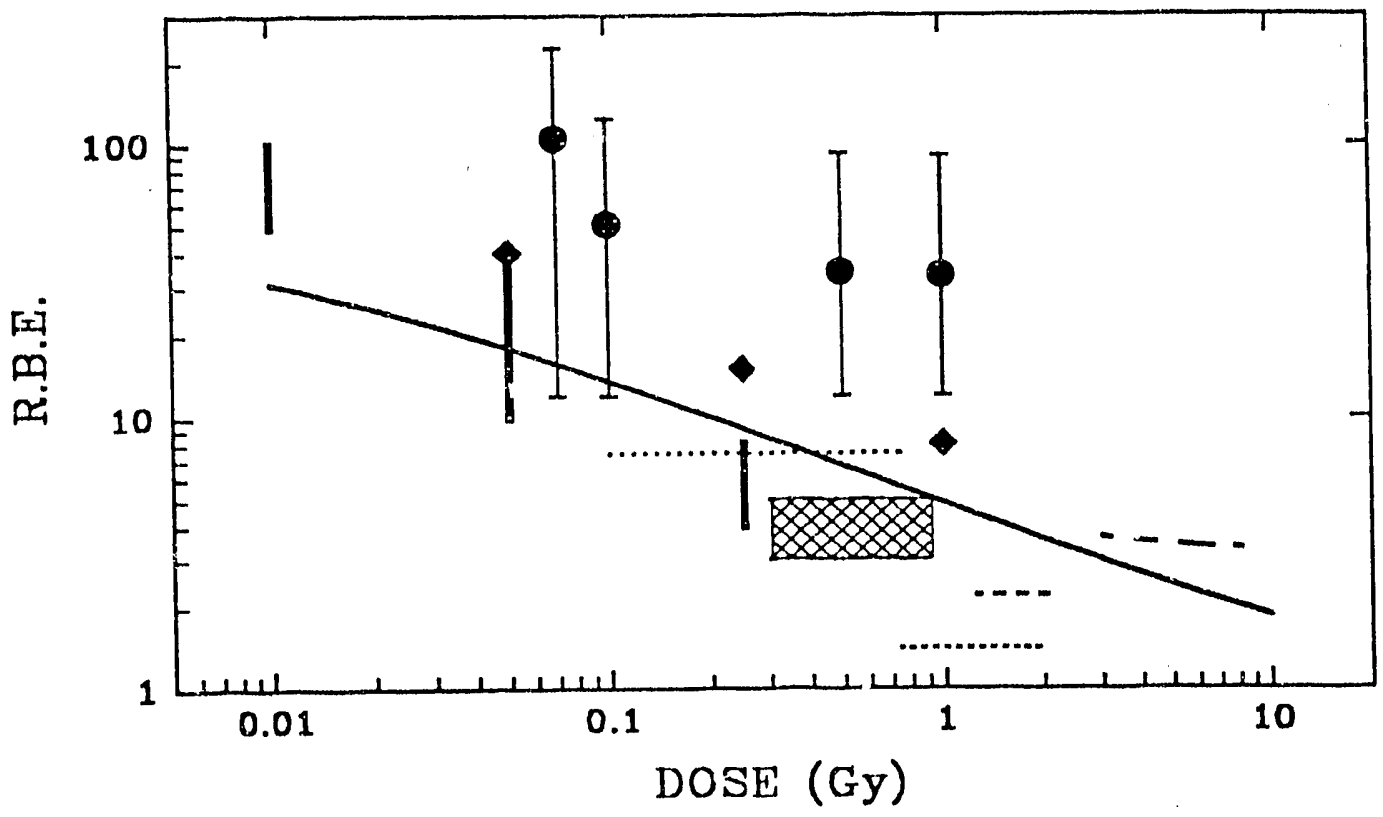


The various points are from the following data:

Otake and Schull (1990), neutrons, humans;
(1984), argon, rats; 1 Brenner et al. (1991), argon, rats; Merriam et al.
argon, mice; - Keng et al. (1982), argon rabbits; -... based on Riley et al. (1991),
neutrons, rats;
data using the linear-quadratic formalism. At intermediate doses it can be approximated as RBE
$=\left(25 / \mathrm{D}_{\mathrm{A}}\right)^{1 / 2}$. The broad confidence limits in the Otake and Schull data reflect uncertainties
regarding dose estimates and model fitting, however, it is in accord with the human data. The
average LET for the neutrons was $\sim 80 \mathrm{keV} / \mu \mathrm{m}$ and the ions $\sim 88 \mathrm{keV} / \mu \mathrm{m}$.

To test whether or not the experimentabfindings for neutrons fall in line with the Otake and Schull estimate and our previous studies using heavy ions, we are in the second year of a life span follow-up with animals irradiated with $435 \mathrm{keV}$ monoenergetic neutrons. To minimize the powerfully modulating effect of age on the cataractogenic process the animals were a very specific age at the time of the irradiation (28 days \pm 1 day). Eight groups of 20 animals each were irradiated with neutrons at the RARAF facility of Columbia University. The animals were divided into dose groups of $0.2,1,5$, and 25 cGy administered as acute doses or in four exposures given at three hour intervals \pm one minute. As indicated, the rat eyes were exposed to $435 \mathrm{KeV}$ neutrons produced by the $\mathrm{T}(\mathrm{p}, \mathrm{n}){ }^{3} \mathrm{He}$ reaction. They were irradiated, two rats at a time, precisely positioned at an angle of $120^{\circ}$ upstream from the target at a distance of $9.4 \mathrm{~cm}$. Specially designed and fabricated jigs guaranteed a position accuracy which allows for no more than a $\pm 5 \%$ deviation in energy and dose between animals. In addition to the neutron exposed rats one group of 20 animals were used as an irradiated control, half of which were employed as sentinels for any possible complicating pathogens which might arise in the colony. These are compared to animals which had been $\mathrm{x}$-irradiated with $0.5,1,2,4,7$ and $10 \mathrm{~Gy}$ of $250 \mathrm{kVp}$ X-rays. Because the doses in the case of neutrons are extremely low as regards cataractogenesis we did not anticipate opacification to commence until the current year. During the course of our follow-up the animals have been examined by conventional slit lamp analysis and Scheimpflug slit lamp imaging using the Zeiss system. The latter methodology is a fully objective technique which provides a permanent record of the cataract which develops. However, only now are we able to incorporate the Scheimpflug system into the study inasmuch as cataracts are just underway. We will also employ the Oxford retroillumination camera, an instrument which provides a standardized photographic based image allowing spatial quantification of the degree of lens involvement. This too is just now beginning to be utilized. The following table lists the mean cataract stage thus far for the various groups. 


\begin{tabular}{lclc} 
Neutrons & 0 & $0.13 \pm 0.04$ & - \\
$(435 \mathrm{keV})$ & 0.2 & $0.20 \pm 0.03$ & $0.31 \pm 0.04$ \\
& 1 & $0.30 \pm 0.03$ & $0.36 \pm 0.05$ \\
& 5 & $0.37 \pm 0.04$ & $0.53 \pm 0.05$ \\
X-rays & 25 & $0.76 \pm 0.04$ & $1.13 \pm 0.06$ \\
$(250 \mathrm{kVp})$ & 0 & $0.14 \pm 0.04$ & - \\
& 50 & $0.19 \pm 0.04$ & - \\
& 100 & $0.33 \pm 0.04$ & - \\
& 200 & $0.50 \pm 0.04$ & $0.29 \pm 0.04$ \\
& 400 & $1.2 \pm 0.07$ & $0.60 \pm 0.04$ \\
& 700 & $2.5 \pm 0$ & - \\
\multicolumn{2}{c}{$=$ fractionated protocols were not used at these dose levels }
\end{tabular}

While the fractionation in the X-ray group demonstrates a clear sparing effect, even in terms of onset, no such sparing has been observed with the neutron. In fact while the data is just now beginning to accrue an enhancement of effect is suggested. Our previous experience with heavy ions led us to believe that enhancement, in terms of cataract onset, is less likely to be observed than is an exacerbation of the progression of the cataract. In any case, the study continues and we are enthusiastic as to its promise and successful completion in the upcoming third and final year of the proposal.

In addition to the ocular assays, on a monthly basis the animals are weighed and examined for tumor development or other pathologies. Blood samples are drawn from the sentinels for monitoring any potential pathogens which would compromise the longevity of the colony.

Under the umbrella of the current funding we have taken advantage of the avail vility of material generated in studies supported by the DOE, not directly associated with our laboratory but with relevance to our goals. One such study, the Harderian gland tumorigenesis grant (DEAC03-76SF-0098) under the aegis of Dr. Edwin Alpin at the Lawrence Berkeley Laboratory is primarily aimed at determining the effects of high-LET radiation on a model for radiation tumor development. Dr. Alpen and his colleagues have generously allowed us access to the animals for cataract analysis and, when sacrificed, have preserved the eye tissues for our cytopathological follow-ups. This has resulted in a paper (Worgul et al, 1989) already published and another one which is in preparation. The studies, fundamentally, support our findings in the rat, although in this case the mo'sse model is utilized. By providing access to eyes irradiated with a host of particle species with LET's ranging from $25 \mathrm{keV}$ to well over $400 \mathrm{keV} / \mu \mathrm{m}$ our data base has increased 
allowing us to generate an appreciation of the possibilities in the response of the system to a myriad of particle energy and types. Perhaps the most exciting result of these studies is the finding that the dose response curve for cataractogenesis mirror that for Harderian gland tumor development, providing additional evidence for the proported genotoxic basis of radiation cataractogenesis. These data also provide the first evidence of damage to the lens being reflected in radiation damage to a tissue less amenable to ready monitoring.

\section{Effort}

The principal investigator, Basil V. Worgul, is overseeing the continued follow-up of this study and is conducting preliminary analysis of the data as it is generated. A portion of the principle investigators time allowance (10\%) also goes to slit lamp observations for the purposes of validation and cross referencing. We are currently in the follow-up mode wherein the animals are examined on a biweekly basis. This has required Dr. Medvedovsky's effort of at least $20 \%$ in its execution. There has been no change in the overall protocol and we anticipate none to occur. The present effort will remain as outlined in our proposals.

According to our veterinary staff the animals are thus far healthy, pathogen-free and should survive the expected median life span of 30 to 36 months.

At the conclusion of the study the animals which remain will be sacrificed and their tissue subject to cytopathological analysis

\section{Publications.}

Peer Revieweci papers published with at least partial support from the DOE April, 1991 - Present

Medvedovsky C and Worgul BV (1991). Neutron effects on the lens. Rad. Res. 128:103-110. Brenner DJ, Medvedovsky C, Huang Y, Merriam GR, Jr. and Worgul BV (1991). Accelerated heavy particles and the lens VI: RBE studies at low doses. Rad. Res. 128:73-81. Worgul BV, David J, Odrich S., Merriam GR, Jr., Medvedovsky C, Merriam JC, Trokel SL and Geard $C R$ (1991). Evidence of genotoxic damage in human cataractous lenses. Mutagenesis $\underline{6}: 495-499$.

Koniarek J and Worgul BV (In Press). Do heavy ions cause microlesions in cell membranes? Adv. in Space Res. 


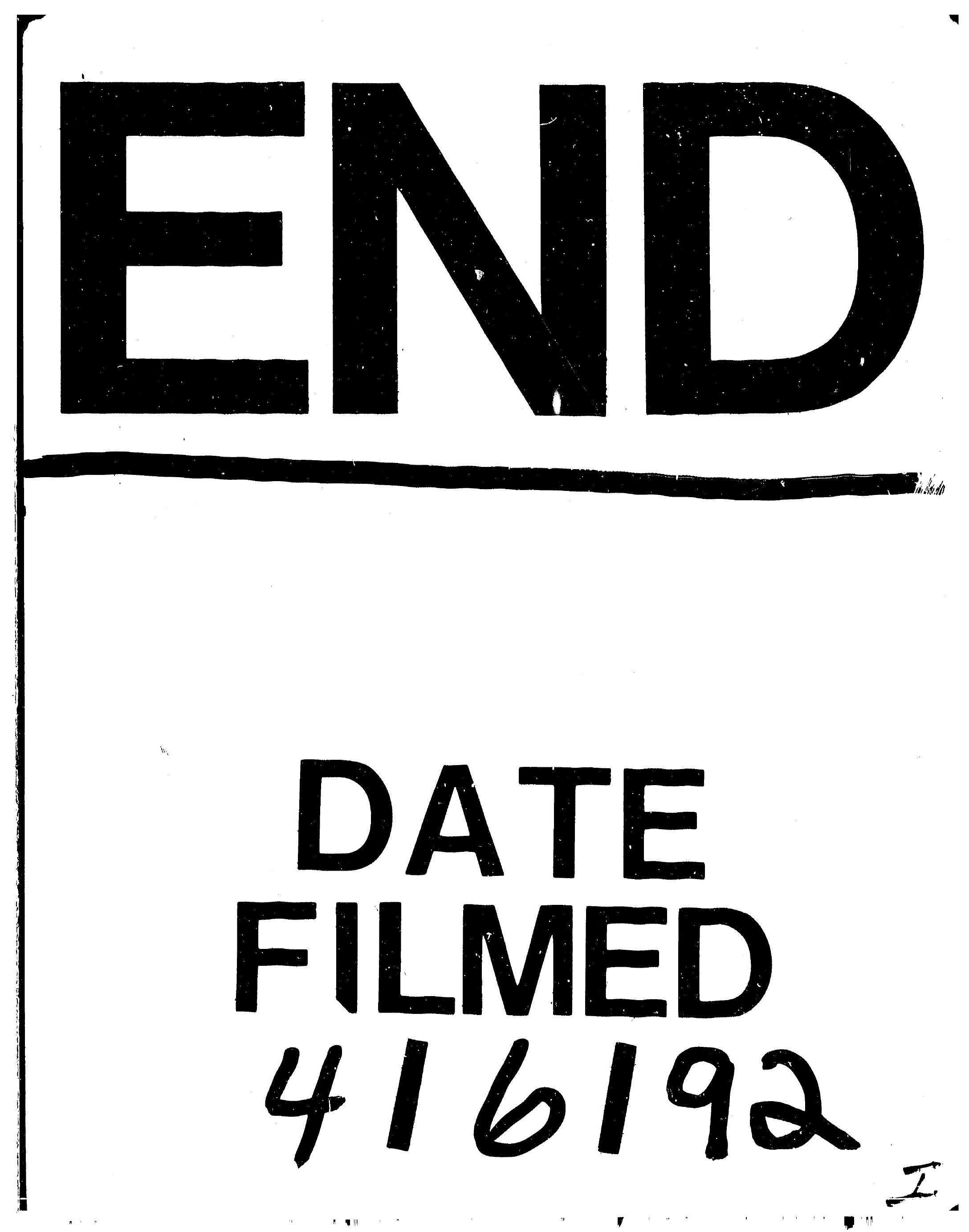




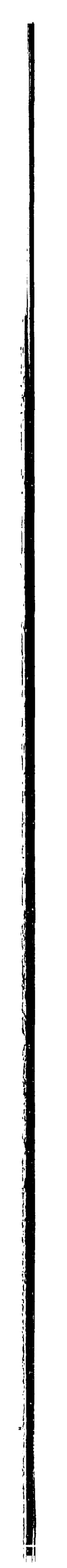

